$\left.p^{\prime}\right)$ and the $h$-index. Using the Spearman correlation coefficient, a value of 0.999 is obtained between $p$ and $p^{\prime}$, indicating an almost perfect correlation between these two measures. Thus, they produce the same general conclusions. In turn, the correlation coefficient between $p^{\prime}$ and $h$ is 0.942 . Despite this high correlation between $p^{\prime}$ and $h$, it is important to highlight that the level of granularity of $p^{\prime}$ (and $p$ ) is much higher than in the case of the $h$-index.

Bibliometric measures are critical inputs for many decisions in universities and research units, including hiring, promotions, research funds allocation and rankings. In this context, the evaluation of performance is critical. In this study, we propose a small adjustment in the $p$ index advanced by Prathap ${ }^{7,8}$. The main advantage of our measure is that it can be obtained directly from the table presented here, without any calculations. This is an important merit since it improves access and transparency in the bibliometric assessment of publication performance.

1. Perry, M. and Reny, P., How to count citations if you must. Am. Econ. Rev., 2016, 106, 2722-2741.

2. Barnes, C., The $h$-index debate: an introduction for librarians. J. Acad. Libr., 2017, 43, 487-494.

3. Hamermesh, D., Citations in economics: measurement, uses, and impacts. J. Econ. Lit., 2018, 56, 115-156.

4. Schreiber, M., A skeptical view on the Hirsch index and its predictive power. Phys. Scripta., 2018, 93, 102501.

5. Hicks, D., Wouters, P., Waltman, L., De Rijcke, S. and Rafols, I., Bibliometrics: the Leiden manifesto for research metrics. Nature News, 2015, 520, 429 .

6. Hirsch, J., An index to quantify an individual's scientific research output. Proc. Natl. Acad. Sci. USA, 2005, 102, 16569-16572.

7. Prathap, G., Is there a place for a mock $h$-index? Scientometrics, 2010, 84, 153-165.

8. Prathap, G., The 100 most prolific economists using the $p$-index. Scientometrics, 2010, 84, 167-172.

9. Anderson, T., Hankin, R. and Killworth, P., Beyond the Durfee square: enhancing the $h$-index to score total publication output. Scientometrics, 2008, 76, 577-588.

10. Prathap, G., The zynergy-index and the formula for the $h$-index. J. Assoc. Inf. Sci. Technol., 2014, 65, 426-427.

ACKNOWLEDGEMENTS. This work was supported by the Fundação para a Ciência e a Tecnologia under Grant UID/GES/ 00315/2019. We thank Gangan Prathap for useful discussions.

Received 17 August 2019; accepted 21 August 2019

doi: $10.18520 / \mathrm{cs} / \mathrm{v} 117 / \mathrm{i} 11 / 1872-1874$

\section{Comparison of parametric and non-parametric methods for chlorophyll estimation based on high-resolution UAV imagery}

\author{
Gaurav Singhal $^{1,2}$, Babankumar Bansod ${ }^{1,2, *}$, \\ Lini Mathew ${ }^{3}$, Jonali Goswami ${ }^{4}$, \\ B. U. Choudhury ${ }^{5}$ and P. L. N. Raju ${ }^{4}$ \\ ${ }^{1}$ CSIR-Central Scientific Instruments Organization, \\ Chandigarh 160 030, India \\ ${ }^{2}$ Academy of Scientific and Innovative Research, \\ Ghaziabad 201002 , India \\ ${ }^{3}$ National Institute of Technical Teachers, Training and Research, \\ Chandigarh 160 019, India \\ ${ }^{4}$ North East Space Application Centre, Barapani 793 103, India \\ ${ }^{5}$ ICAR Research Complex for NEH Region, Umiam 793 103, India
}

The present study provides a systematic comparison of parametric and non-parametric retrieval methods using high-resolution data provided by the unmanned aerial vehicle (UAV). We used turmeric crop reflectance data to evaluate the vegetation index (VI)-based parametric methods and compared them with linear and nonlinear non-parametric methods to build a rigorous LCC estimation model. The study demonstrates that the best-performing VI was the normalized green red difference index (GNRDI), with $R^{2}=0.68$, RMSE $=0.13$ and high processing speed of $0.08 \mathrm{~s}$. With regard to non-parametric methods, almost all methods outperformed their parametric counterparts. Particularly, methods such as random forest (RF) and kernel ridge regression (KRR) showed the best performance characterized by $R^{2}>0.72$ and RMSE $\leq 0.12 \mathrm{mg} / \mathrm{g}$ of fresh leaf weight. These nonparametric methods possessed the benefit of total spectral information utilization and enabled robust, non-linear relationship between the predictor and target variables, but computational complexity is a major drawback.

Keywords: Chlorophyll, machine learning, unmanned aerial vehicle, vegetation index.

BEING the most important pigment of all photosynthetic cells, variations in leaf chlorophyll concentration (LCC) are an indicator of crop growth and stress status and help in estimating biomass and yield ${ }^{1-3}$. Chlorophyll is best described by its absorption in the red band (600-720 nm) and major reflection from green and NIR wavebands ${ }^{4}$.

Remote sensing sensors play an important role in crop health monitoring in terms of large coverage area and fast estimates of crop biophysical and biochemical parameters such as LCC. Remote sensing satellites are capable of providing a vast coverage, but their potential is limited by poor resolution and cloud cover. Flying at low altitude

*For correspondence. (e-mail: csiobabankumar@gmail.com) 
and beneath the clouds, UAV overcomes these limitations and provides more flexibility ${ }^{5}$. It can provide high spatial and temporal resolution data. The high resolution allows precise estimation of plant biophysical parameters such as LCC and leaf nitrogen content from multispectral (MS) images of a field.

Several non-destructive methods, including parametric and non-parametric approaches to estimate the LCC have been proposed. VI-based parametric approach has been fascinating in the remote-sensing community to estimate the crop biophysical parameters such as $\mathrm{LCC}^{6-8}$. VI can extract more than $90 \%$ of spectral information of the vegetation. Index normalization typically upgrades the interpretability of plant properties by diminishing the effect of non-photosynthetic plant components, for example, soil background ${ }^{9}$, but they are prone to show poor model performance because of some limitations ${ }^{10}$ such as atmospheric effects and canopy structural characteristics $^{9,11}$. In addition, VI regression models are likely to show unstable performance when imposed to MS images differing from the simulated ones.

Another set of methods to retrieve the LCC comprises non-parametric methods including multiple linear regression (MLR), principal component regression (PCR), partial least square regression (PLSR), support vector machine (SVM), relevance vector machine (RVM), random forest (RF) and kernel ridge regression (KRR). Literature review unfolds that these methods utilize full spectral data, avoids collinearity and provides a robust relationship between predictor variables and the variables of interest ${ }^{12}$. So far, only a few studies have been carried out to estimate the LCC of turmeric based on highresolution UAV imagery ${ }^{1,13-15}$. Therefore, the explicit objective of this study includes the assessment of these retrieval methods for LCC estimation of turmeric crop using UAV based high-resolution multispectral imagery.

The UAV used for this field study was a Hex-copter from DJI (www.DJI.com). It was equipped with multispectral sensor (parrot sequoia), consisting of four bands, i.e. green (spectral bandwidth of 530-570 nm, centred at $550 \mathrm{~nm}), \quad(640-680 \mathrm{~nm}, \quad 660 \mathrm{~nm}), \quad$ Red-Edge $(730$ $740 \mathrm{~nm}, 735 \mathrm{~nm})$ and NIR $(770-810 \mathrm{~nm}, 790 \mathrm{~nm})$. The spatial resolution achieved was $2 \mathrm{~cm}$ at an elevation of $30 \mathrm{~m}$. The image capture rate was one frame per second. UAV flights were carried out in ambient light conditions within the time frame of 11:30 a.m. and 1:00 p.m. (IST). Pix-4D image processing software was used to perform radiometric calibrations, generating orthomosaics and reflectance maps from UAV images. A white reference (calibration) panel was used to calibrate and correct the acquired UAV data into surface reflectance based on the illumination condition and properties of sensors. For more information about the data collection, please refer to the previous study ${ }^{16}$.

For this study, flight's chlorophyll and reflectance data were collected from the research farms of Indian Council of Agricultural Research (ICAR) for North East hilly region at Umiam (Shillong, Meghalaya) located at $25^{\circ} 41^{\prime} \mathrm{N}$ lat, $91^{\circ} 55^{\prime} \mathrm{E}$ long. Turmeric was grown over 20 equal-sized plots with 35-40 plants in each plot. Simultaneously with UAV flight, the SPAD-502 chlorophyll meter readings were taken from plant samples. Six hundred reflectance and SPAD values from 20 plots (30 samples per plots) were available to develop the model. The SPAD-502 chlorophyll metre measurements exhibited an exemplary agreement with laboratory-measured leaf chlorophyll concentration (LCC) values in terms of regression coefficient $\left(R^{2}=0.92\right)$, which was reported earlier ${ }^{16}$. These spectral reflectance values of all bands were combined with LCC measurements to calibrate and validate the LCC estimation model.

The parametric methods used in this study were based on the calculation of VIs from reflectance maps. A total of 11 two and three bands indices (Table 1) were formulated to regress them against the LCC using ordinary least square method.

Non-parametric methods were subdivided into linear and non-linear methods; former include MLR, PCR and PLSR. These methods are fascinating because of their fast speed and develop a linear relationship between the predictor and target variables. Methods like PCR and PLSR often remove the collinearity with dimensionality reduction approach ${ }^{25}$. Non-linear non-parametric methods are also known as machine learning methods. These include SVR, RVR, RF and KRR, which are comprehensively described in the following section.

Support vector regression (SVR) combined with a kernel function, maps the training samples into a higher dimensional feature space, which is nonlinearly related to the original space ${ }^{25}$. It is used for classification purpose when the number of features is larger compared to the number of data points in the dataset. SVR using radial basis function (RBF) kernel requires the tuning of two hyper-parameters, i.e. the cost-penalty parameter $\mathrm{C}$ and the standard deviation $(\sigma)$. C and $\sigma$ parameters directly control the model's performance. For higher values of $\mathrm{C}$, the model becomes intolerable for large errors, and is more prone to overfitting, whereas, for smaller values of $\mathrm{C}$, the model shows rigidness and shows underfitting.

The relevance vector machine (RVM) is a wellrecognized machine learning algorithm that uses a Bayesian framework and follows a probabilistic sparse kernel model for classification and regression ${ }^{26}$. This approach delivers a statistical relationship based on only a smaller number of training samples, called relevance vectors. Sparsity is achieved because the posterior distributions of weights are sharply peaked around zero.

The kernel ridge regression (KRR) is essentially regularized SVM. A regularization parameter $(\lambda)$ is added to the cost function to control the values of the anticipated parameters, forms the closed from solutions ${ }^{27}$ and removes overfitting. The KRR method ${ }^{28}$ solves the cost 


\section{RESEARCH COMMUNICATIONS}

Table 1. Vegetation index used in this study

\begin{tabular}{|c|c|c|}
\hline Vegetation index (Acronym) & Formula & Reference \\
\hline Normalized difference vegetation index (NDVI) & $\frac{\mathrm{NIR}-\mathrm{RED}}{\mathrm{NIR}+\mathrm{RED}}$ & 17 \\
\hline Normalized green red difference index (NGRDI) & $\frac{\text { GREEN - RED }}{\text { GREEN + RED }}$ & 18 \\
\hline Normalized difference red-edge index (NDRE) & $\frac{\text { NIR - REDEDGE }}{\text { NIR + REDEDGE }}$ & 19 \\
\hline Green normalized difference vegetation index (GNDVI) & $\frac{\text { NIR - GREEN }}{\text { NIR + GREEN }}$ & 13 \\
\hline Chlorophyll vegetation index (CVI) & $\mathrm{NIR} * \frac{\mathrm{RED}}{\mathrm{GREEN}^{2}}$ & 20 \\
\hline Soil adjusted vegetation index (SAVI) & $\frac{\mathrm{NIR}-\mathrm{RED}}{\mathrm{NIR}+\mathrm{RED}}+0.5$ & 20 \\
\hline Simple ratio vegetation index (SRVI) & $\frac{\text { NIR }}{\text { GREEN }}$ & 2 \\
\hline Chlorophyll green index (Clgreen) & $\frac{\mathrm{NIR}}{\mathrm{GREEN}}-1$ & 22 \\
\hline Chlorophyll red-edge index (Clrededge) & $\frac{\mathrm{NIR}}{\mathrm{RED}-\mathrm{EDGE}}-1$ & 20 \\
\hline Modified chlorophyll absorption ratio index (MCARI) & {$[($ RedEdge - Red $)-0.2($ RedEdge - Green $)($ RedEdge/Red $)]$} & 23 \\
\hline Transformed chlorophyll absorption ratio index (TCARI) & $3[($ RedEdge - Red $)-0.2($ RedEdge - Green $)($ RedEdge $/$ Red $)]$ & 24 \\
\hline
\end{tabular}

Table 2. Descriptive statistics of individual bands and spectral indexes derived from UAV images

\begin{tabular}{llcl}
\hline $\begin{array}{l}\text { Spectral } \\
\text { index }\end{array}$ & Range & $\begin{array}{c}\text { Mean, } \\
\text { standard deviation }\end{array}$ & $\begin{array}{c}\text { Correlation } \\
\text { coefficient }\end{array}$ \\
\hline SR & $8.57-20.79$ & $13.28,2.47$ & 0.64 \\
NDVI & $0.028-0.891$ & $0.658,0.151$ & 0.78 \\
NDRE & $0.07-0.32$ & $0.15,0.03$ & 0.28 \\
NGRDI & $0.290-0.515$ & $0.47,0.04$ & 0.83 \\
GNDVI & $0.52-0.74$ & $0.64,0.04$ & 0.68 \\
SAVI & $0.009-0.469$ & $0.302,0.086$ & 0.734 \\
CVI & $1.13-2.67$ & $1.69,0.33$ & 0.48 \\
CI-Green & $2.23-5.88$ & $3.71,0.73$ & 0.64 \\
CI-RedEdge & $0.154-0.94$ & $0.35,0.09$ & 0.203 \\
MCARI & $-0.498-0.097$ & $-0.183,0.112$ & 0.26 \\
TCARI & $0.22-0.90$ & $0.51,0.123$ & 0.35 \\
\hline
\end{tabular}

function by transforming the data-points in to a higher dimension space. In this study, the KRR hyperparameters were tuned via $K$-fold cross-validation.

Random forest (RF) is an ensemble learning approach which consists of a number of decision trees. The response of RF is the mean prediction of individual trees after proper training. It has been used for building classification and regression models in several remote sensing studies $^{29,30}$. The best prediction model was obtained by tuning hyperparameter mtry, which represents the number of random variables at each node split.

The following radial basis function was utilized in all used kernel based non-parametric methods for model building
Radial basis function $(\mathrm{RBF})=\mathrm{e}^{\left(\left\|x-x^{\prime}\right\|^{2}\right) / 2 \sigma^{2}}$,

where $x$ and $x^{\prime}$ are two samples representing feature vectors. $\sigma$ is the tunable parameter for RBF kernel. It directly affects model performance.

It ought to be noticed that the whole data set containing the predictor variables and LCC was divided into training and testing dataset in a ratio of $70: 30$ for model development and validation. $K$-fold cross-validation $(K=10)$ was used for hyper-parameters tuning, where the training data was further divided into training and validation dataset at $90: 10$ ratios for $K$ times.

We first concentrated on assessing the connection between LCC and multispectral VIs based on the coefficient of determination $R^{2}$ and RMSE. Later, we evaluated nonparametric methods and contrasted the outcomes with optimal VIs based on $R^{2}$ and RMSE.

Relationship between LCC and 11 different two-band vegetation indices was recognized using the ordinary least square method. Table 2 represents the average correlation coefficient $(R)$ values between multispectral VIs and LCC using $K$-fold cross-validation of 100 iterations. Results elucidate that both NGRDI and NDVI are the best performing vegetation indices $(R=0.83$ and 0.78$)$ respectively, outperforming other indices. These results agree with earlier studies ${ }^{20,31,32}$, where NGRDI and NDVI were found as efficient methods for LCC estimation in crop health monitoring. It is noteworthy that both optimal indices consisted of the red band $(660 \mathrm{~nm})$. Despite the fact that outcomes of adequate accuracy were acquired in this study utilizing this basic model, various disadvantages 
remain, such as saturation of NDVI (saturated NDVI value $=0.89$ ) at high LCC values ${ }^{33}$. Moreover, it is effectively influenced by the growth stage, and the crucial information may be absent at other spectral wavebands.

All non-parametric methods were evaluated in the MATLAB-based automated radiative transfer models operator (ARTMO) toolbox (freely available at https:// artmotoolbox.com) to build a rigorous LCC estimation model. Table 3 shows that all non-parametric methods outperformed their parametric counterparts and KRR proved to be the best performing method with RMSE = 0.10 and $R^{2}=0.7452$. Among non-parametric methods, linear methods showed extremely fast results but with less prediction accuracy. PCR and PLSR show almost similar results with $R^{2}=0.7$ and $\mathrm{RMSE}=0.128 \mathrm{mg} / \mathrm{g}$ for test data set. These similar results were achieved because of a smaller number of predictor variables available in the analysis. Although MLR, PCR and PLSR have been used in previous studies to model the chlorophyll concentration values in the present study, they were outperformed by their more flexible non-linear counter parts and could not give satisfactory statistical performance. Therefore, we have limited their discussion in this study. The evaluation and statistical performance of non-linear non-parametric methods are discussed in the following section.

For SVR model, the kernel parameters $\sigma$ and $\mathrm{C}$ were tuned using $K$-fold cross-validation and the optimum parameters were selected based on minimum RMSE. $\sigma$ was tuned between 0.10 and 64 on the logarithmic $(\log 2)$ scale and a cost parameter $C=1.8$ was finalized to train the model with minimum RMSE and maximum $R^{2}$. The increment in $C$ parameter increased the error, which led to under-fitting of the model. Table 3 shows the RMSE and $R^{2}$ values for both training and testing dataset. We found that it showed some over fitting and could not perform well for pixels related to poor vegetation and nonvegetation while distributing the LCC values.

The random forest model was run with hyperparameter value $(m$ try $=2)$ as the optimum value that satisfies the theoretically recommended (one-third of total input variables) value of mtry. We observed that RF performed most accurately for the training dataset among all models but poor for the test dataset, i.e. clear overfitting. The

Table 3. Performance table of different non-parametric regression algorithms in LCC estimation

\begin{tabular}{lcccc}
\hline Method & $\begin{array}{c}R^{2} \\
\text { (training) }\end{array}$ & $\begin{array}{c}\text { RMSE } \\
(\mathrm{mg} / \mathrm{g})\end{array}$ & $\begin{array}{c}R^{2} \\
\text { (validation) }\end{array}$ & $\begin{array}{c}\text { RMSE } \\
(\mathrm{mg} / \mathrm{g})\end{array}$ \\
\hline MLR & 0.72 & 0.12 & 0.65 & 0.15 \\
PCR & 0.80 & 0.095 & 0.70 & 0.13 \\
PLSR & 0.80 & 0.095 & 0.70 & 0.13 \\
SVR & 0.82 & 0.090 & 0.70 & 0.13 \\
RF & 0.86 & 0.075 & 0.72 & 0.12 \\
RVR & 0.812 & 0.085 & 0.71 & 0.12 \\
KRR & 0.832 & 0.090 & 0.74 & 0.10 \\
\hline
\end{tabular}

over fitting of this model is evident from the large difference between training and testing statistics (Table 3).

The RVR method showed some improvements in RMSE and $R^{2}$ with its counterpart SVR because of its Bayesian framework approach. The only advantage of RVR is the avoidance of free hyperparameters setting which requires cross-validation for their optimization. The large processing time of RVR $(16.35 \mathrm{sec})$ limits this approach to integrate with real-time crop health monitoring systems.

The KRR method proved to be the best performing nonlinear regression method among all methods yielding, an RMSE of $0.10 \mathrm{mg} / \mathrm{g}, R^{2}$ of 0.7452 and extremely fast processing speed of 1.434 seconds as the most robust outcomes. This approach clearly distributed the LCC values spatially throughout the turmeric plots. This approach set a nonlinear relationship between LCC and spectral data and had the advantage of handling a large number of datasets with high speed and accuracy. The results of KRR achieved in this study corroborate with previous studies ${ }^{12,34}$.

Table 3 shows the $R^{2}$ and RMSE values for all machine learning methods with respect to training and testing dataset. A larger difference between training and testing dataset represents the high-performance difference between them. KRR showed the least difference between the two sets of data as this method is less sensitive to the outliers. Although the RF is also not sensitive to outliers, it could not perform well with the test data with the smaller number of samples. Overall, the KRR model performed best with both the datasets and therefore, can be used for generating the chlorophyll maps ${ }^{16}$.

Figure $1 a$ presents scatter plots of estimated versus measured LCC values of all samples derived from the best performing non-parametric KRR algorithm. From the scatter plot, it can be deduced that KRR is superior to NGRDI in terms of model performance as the estimated LCC values are closer to $1: 1$ line than the optimal VI scatter plot. Notwithstanding its better execution, KRR is substantially more computationally complex than NGRDI due to optimization steps.

We also measured the importance of predictor variables using the Gaussian process regression (GPR) ${ }^{35}$. GPR band assessment technique was performed in the Artmo toolbox in MATLAB and the best contributing band was detected on the basis of sigma value as shown in Figure $1 b$. Since lower sigma value leads to higher information, the red $(660 \mathrm{~nm})$ and green $(550 \mathrm{~nm})$ bands hold the biggest quality impact on LCC estimation, regardless of distinctive methodologies utilized for LCC estimation. These results justify the selected vegetation indices, which show the dominance of red band at least for this study site and specific crop.

Although several studies have endeavoured to utilize UAV-based MS images to estimate the LCC status, the datasets were not sufficient enough to get the satisfactory 

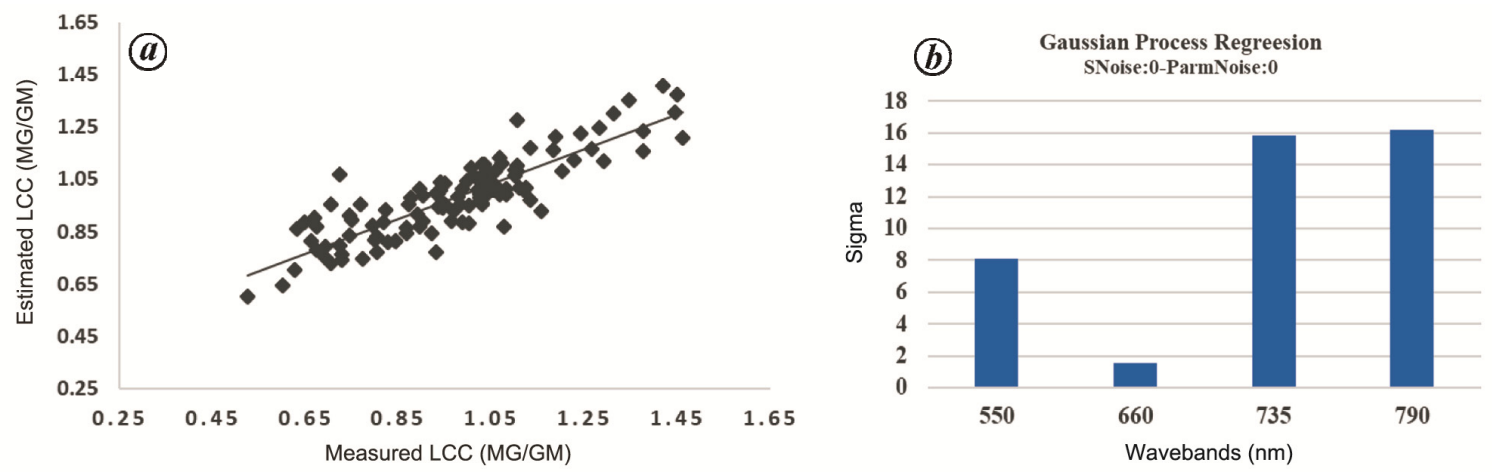

Figure 1. $\boldsymbol{a}$, Measured versus estimated LCC values derived using the KRR algorithm. $\boldsymbol{b}$, Spectral bands importance order based on GPR.

LCC estimation accuracy. Conversely, the outcomes of this analysis demonstrate that GNDRI created higher assessments, while the non-parametric KRR technique prompted a higher level of accuracy under various conditions. These outcomes propose that UAV-based MS image give a promising methodology that can be integrated with crop health status monitoring. Moreover, even after getting higher order estimation accuracy, the built-up LCC estimation model in any case should be tried with another crop and ecological site with distinct conditions.

In this study, parametric and non-parametric modelling techniques were compared to estimate the LCC using UAV-based high-resolution images. Results demonstrated that NGRDI and NDVI are highly correlated with LCC, but show some limitations like saturation effect at higher LCC values. The non-parametric techniques outperformed parametric techniques in terms of model performance. Furthermore, among nonparametric schemes, techniques like RF and KRR showed the best performance. Linear non-parametric approach was outperformed by its nonlinear partners in this study. The extremely fast speed recommends them to be integrated with the crop health status monitoring systems.

Disclosure statement: Authors declare no conflict of interest to disclose.

1. Kanning, M., Kühling, I., Trautz, D. and Jarmer, T., Highresolution UAV-based hyperspectral imagery for LAI and chlorophyll estimations from wheat for yield prediction. Remote Sensing, 2018, 10, 2000.

2. Pearson, R. L. and Miller, L. D., Remote mapping of standing crop biomass for estimation of the productivity of the short grass prairie. Remote Sensing Environ., 1972, VIII, 1355.

3. Pérez-Patricio, M. et al., Optical method for estimating the chlorophyll contents in plant leaves. Sensors, 2018, 18, 650.

4. Thomas, J. and Gausman, H., Leaf reflectance versus leaf chlorophyll and carotenoid concentrations for eight crops 1. Agron. J., 1977, 69, 799-802.

5. Aasen, H., Burkart, A., Bolten, A. and Bareth, G., Generating 3D hyperspectral information with lightweight UAV snapshot cameras for vegetation monitoring: from camera calibration to quality assurance. ISPRS J. Photogramm., 2015, 108, 245-259.

6. Broge, N. H. and Leblanc, E., Comparing prediction power and stability of broadband and hyperspectral vegetation indices for estimation of green leaf area index and canopy chlorophyll density. Remote Sensing Environ., 2001, 76, 156-172.

7. Haboudane, D., Tremblay, N., Miller, J. R. and Vigneault, P., Remote estimation of crop chlorophyll content using spectral indices derived from hyperspectral data. IEEET Geosci. Remote Sensing, 2008, 46, 423-437.

8. Hunt, E. R., Daughtry, C., Eitel, J. U. and Long, D. S., Remote sensing leaf chlorophyll content using a visible band index. Agron. $J ., 2011,103,1090-1099$.

9. Baret, F. and Guyot, G., Potentials and limits of vegetation indices for LAI and APAR assessment. Remote Sensing Environ., 1991, 35, 161-173.

10. Baret, F., Guyot, G. and Major, D., TSAVI: a vegetation index which minimizes soil brightness effects on LAI and APAR estimation. In 12th Canadian Symposium on Remote Sensing Geoscience and Remote Sensing Symposium, Vancouver, Canada, 1989, pp. $1355-1358$

11. Bannari, A., Morin, D., Bonn, F. and Huete, A., A review of vegetation indices. Remote Sensing Rev., 1995, 13, 95-120.

12. Caicedo, J. P. R., Verrelst, J., Muñoz-Marí, J., Moreno, J. and Camps-Valls, G., Toward a semiautomatic machine learning retrieval of biophysical parameters. IEEE J. Selected Top. Appl. Earth Observ. Remote Sensing, 2014, 7, 1249-1259.

13. Hunt, E. R., Hively, W. D., Fujikawa, S., Linden, D., Daughtry, C. S. and McCarty, G., Acquisition of NIR-green-blue digital photographs from unmanned aircraft for crop monitoring. Remote Sensing, 2010, 2, 290-305.

14. Roosjen, P. P., Brede, B., Suomalainen, J. M., Bartholomeus, H. M., Kooistra, L. and Clevers, J. G., Improved estimation of leaf area index and leaf chlorophyll content of a potato crop using multi-angle spectral data-potential of unmanned aerial vehicle imagery. Int. J. Appl. Earth Observ. Geoinform., 2018, 66, 14-26.

15. Simic Milas, A., Romanko, M., Reil, P., Abeysinghe, T. and Marambe, A., The importance of leaf area index in mapping chlorophyll content of corn under different agricultural treatments using UAV images. Int. J. Remote Sensing, 2018, 39, 5415-5431.

16. Singhal, G., Bansod, B., Mathew, L., Goswami, J., Choudhury, B. and Raju, P., Estimation of leaf chlorophyll concentration in turmeric (Curcuma longa) using high-resolution unmanned aerial vehicle imagery based on Kernel Ridge Regression. J. Indian Soc. Remote Sensing, 2019, 47, 1-12.

17. Rouse Jr, J. W., Haas, R., Schell, J. and Deering, D., Monitoring vegetation systems in the Great Plains with ERTS. In NASA, 3d ERTS-1 Symposium, Goddard Space Flight Center, 1974, vol. 1, Sect. A, pp. 309-317.

18. Tucker, C. J., Red and photographic infrared linear combinations for monitoring vegetation. Remote Sensing Environ., 1979, 8, $127-150$.

19. Gitelson, A. and Merzlyak, M. N., Quantitative estimation of chlorophyll- $a$ using reflectance spectra: experiments with autumn 
chestnut and maple leaves. J. Photochem. Photobiol. Biol., 1994 22, 247-252.

20. Hunt Jr, E. R., Doraiswamy, P. C., McMurtrey, J. E., Daughtry, C. S., Perry, E. M. and Akhmedov, B., A visible band index for remote sensing leaf chlorophyll content at the canopy scale. Int. $J$. Appl. Earth Observ. Geoinf., 2013, 21, 103-112.

21. Huete, A. R., A soil-adjusted vegetation index (SAVI). Remote Sensing Environ., 1988, 25, 295-309.

22. Gitelson, A. A., Gritz, Y. and Merzlyak, M. N., Relationships between leaf chlorophyll content and spectral reflectance and algorithms for non-destructive chlorophyll assessment in higher plant leaves. J. Plant Physiol., 2003, 160, 271-282.

23. Daughtry, C., Walthall, C., Kim, M., De Colstoun, E. B. and McMurtrey Iii, J., Estimating corn leaf chlorophyll concentration from leaf and canopy reflectance. Remote Sensing Environ., 2000, 74, 229-239.

24. Haboudane, D., Miller, J. R., Tremblay, N., Zarco-Tejada, P. J. and Dextraze, L., Integrated narrow-band vegetation indices for prediction of crop chlorophyll content for application to precision agriculture. Remote Sensing Environ., 2002, 81, 416-426.

25. Verrelst, J. et al., Quantifying vegetation biophysical variables from imaging spectroscopy data: a review on retrieval methods. Surveys Geophys., 2018, 40(3), 1-41.

26. Tipping, M. E. (ed.), Bayesian inference: An introduction to principles and practice in machine learning. In Advanced Lectures on Machine Learning, Springer, 2004, pp. 41-62.

27. Cawley, G. C., Talbot, N. L. and Chapelle, O., Estimating predictive variances with kernel ridge regression. In Machine Learning Challenges Workshop, 2005, pp. 56-77.

28. Saunders, C., Gammerman, A. and Vovk, V., Ridge regression learning algorithm in dual variables. In ICML '98 Proceeding of the Fifteenth International Conference on Machine Learning, 1998, pp. 515-521.

29. Belgiu, M. and Drăguț, L., Random forest in remote sensing: A review of applications and future directions. ISPRS J. Photogramm. Remote Sensing, 2016, 114, 24-31.

30. Walton, J. T., Subpixel urban land cover estimation. Photogramm. Eng. Remote Sensing, 2008, 74, 1213-1222.

31. Gitelson, A. A. and Merzlyak, M. N., Remote sensing of chlorophyll concentration in higher plant leaves. Adv. Space Res., 1998, 22, 689-692.

32. Jones, C. L., Weckler, P., Maness, N. O., Jayasekara, R., Stone, M. and Chrz, D., Remote sensing to estimate chlorophyll concentration in spinach using multi-spectral plant reflectance. Trans. $A S A B E, 2007, \mathbf{5 0}, 2267-2273$.

33. Hunt, E. R., Cavigelli, M., Daughtry, C. S., Mcmurtrey, J. E. and Walthall, C. L., Evaluation of digital photography from model aircraft for remote sensing of crop biomass and nitrogen status. Precis. Agric., 2005, 6, 359-378.

34. Peng, Y., Huang, H., Wang, W., Wu, J. and Wang, X., Rapid detection of chlorophyll content in corn leaves by using least squares-support vector machines and hyperspectral images. $J$. Jiangsu Univ.-Natural Sci. Edn, 2011, 32, 125-174.

35. Verrelst, J., Rivera, J. P., Gitelson, A., Delegido, J., Moreno, J. and Camps-Valls, G., Spectral band selection for vegetation properties retrieval using Gaussian processes regression. Int. J. Appl. Earth Observ. Geoinform., 2016, 52, 554-567.

ACKNOWLEDGEMENTS. The authors thank the UAV team of NESAC (Shillong) and ICAR (Shillong) for their help in carrying out field data collection and analysis. The experimental research was funded by Department of Science and Technology (DST), New Delhi under the project 'Characterization of acid soils under different land uses and its impact on crop growth: a hyperspectral approach' (Grant Number: DST-BDID/01/23/2014 - HSRS dated 18.03.2016).

Received 2 June 2019; revised accepted 24 July 2019

doi: $10.18520 / \mathrm{cs} / \mathrm{v} 117 / \mathrm{i} 11 / 1874-1879$

\section{Fate and transport of microplastics from water sources}

\author{
Monnisha Ganesan ${ }^{1}$, Gobi Nallathambi ${ }^{1, *}$ and \\ Seshachalam Srinivasalu ${ }^{2}$ \\ ${ }^{1}$ Department of Textile Technology, and \\ ${ }^{2}$ Department of Geology, Institute of Ocean Management, \\ Anna University, Chennai 600 025, India
}

Microplastics as environmental pollutants affect surface water and groundwater. Surface water, groundwater and branded drinking water bottles were analysed in and around Chennai, Tamil Nadu, India. The total count of microplastics was found to be 66 particles with fibrous and fragmented shape, colours such as white, blue, green, yellow, pink and black under optical microscope. SEM-EDX-used to study morphology and elemental analysis of microplastics confirmed the presence of heavy metals such as $\mathrm{Cr}$, $\mathrm{Ti}$, Mo, Ba and Ru adhered to their surface. Polyethylene terephthalate and polyamide were confirmed by the presence of functional groups of the polymers by FTIR equipped with attenuated total reflectance.

Keywords: Microplastics, heavy metals, pollution, polyamide, water sources.

PLASTICS are synthetic organic polymers formed by the process of polymerization ${ }^{1}$. The use of plastics has increased worldwide and the annual production is around 322 million metric tonnes ${ }^{2}$. In India, approximately 5.6 million tonnes (mt) of plastic waste is generated annually ${ }^{3}$. Plastic debris which is less than $5 \mathrm{~mm}$ is referred to as microplastic, and categorized as primary and secondary. The main sources of microplastics in the marine environment are land and sea-based litter ${ }^{4}$. Microplastics are a big threat to marine organisms as they are ingested by them. A study was done on the distribution, weathering and chemical characteristics of microplastics on the beaches of Goa, India during the southwest and northeast monsoon seasons ${ }^{4}$. The distribution and characteristics of microplastic pollution along the coast of Chennai, Tamil Nadu (TN), India during pre- and post-flood were also studied $^{5}$. Presence of microplastics and their distribution and characteristics were reported from Rameswaram Coral Island, TN, India ${ }^{6}$, marine water from Kuala Nerus and Kuantan Port in Malaysia $^{7}$, drinking water treatment plants in Germany ${ }^{8}$, freshwater resources like Vembanad lake in Kerala ${ }^{3}$, Huron Lake in Canada ${ }^{9}$, and three urban estuaries in China ${ }^{10}$. Microplastics were also identified in bottled drinking water, because of the packaging materials (polyethylene terephthalate), which are consumed by humans ${ }^{11}$. In India, only a few studies are available on microplastics contamination in sediment samples of

*For correspondence. (e-mail: gobsnn@gmail.com) 